\title{
NOMINALISMUS ALS UNIVERSITÄRE SPEKULATIONSKONTROLLE
}

\author{
Maarten J. F. M. HoEnEN
}

Abstract

This article provides a historico-philosophical profile of late-medieval nominalism. It shows how nominalism placed limitations on the use of logic and natural reason in academic theology. In the first part, the historical meaning and use of the notions 'moderni' and 'nominales' are explored. In the second part, the methodology of the 'moderni' and 'nominales' is investigated, showing how nominalist understanding of logic and natural reason led to a separation of philosophy and theology. Three examples are discussed, taken from commentaries on the Sentences. As these examples show, according to a number of 'moderni' and 'nominales' (Durandus de St Pourçain, William of Ockham, Adam Wodeham, Robert Holkot, Marsilius of Inghen), logic and natural reason are not able to exclude all ambiguities and heresies from theological discourse. Thus logic loses its privileged function in theology as arbiter of true reasoning, clearing the way for the rules of traditional and ordinary speech, the 'vulgus modus loquendi'. Only when logic and reason deal with the natural aspects of creation, do they remain authoritative. When applied to the divine, however, they may easily lead to heresy and error.

\section{Einleitung}

'Nominalismus als universitäre Spekulationskontrolle': Dies ist ein besonderer Titel, der die Phantasie anregt und zum Nachdenken veranlasst, namentlich aufgrund des Ausdrucks 'Spekulationskontrolle' . $\mathrm{Zu}$ meinem eigenen Bedauern muss ich zugeben, dass dieser Titel nicht von mir stammt, sondern von den Organisatoren der Berliner Tagung 2 . Der Titel wirft Fragen auf, und das soll ein Titel ja schließlich leisten.

1. Zu diesem Thema sind in den letzten Jahren eine Reihe von Publikationen erschienen, von denen besonders hervorzuheben sind J. M. M. H. THIJssen, Censure and Heresy at the University of Paris, 1200-1400, Philadelphia 1998, und L. BIANCHI, Censure et Liberté Intellectuelle à l'Université de Paris (XIIIe-XIVe Siècles), Paris 1999.

2. Gründungstagung der GPMR, Imagination und Institution (Berlin, 14.-16.10.2004), organisiert von Wilhelm Schmidt-Biggemann und Sebastian Lalla. 
Ich möchte daher auch mit einer Frage beginnen, und zwar mit der nächstliegenden: Was heißt es, wenn man vom 'Nominalismus' als universitärer Spekulationskontrolle spricht? Will man damit sagen, dass der Nominalismus 'mehrere' Gesichter hat, dass eines dieser Gesichter die universitäre Spekulationskontrolle ist und man auf dieses Gesicht die Aufmerksamkeit richten möchte? Oder will man damit sagen, dass das 'Kennzeichen' des Nominalismus die universitäre Spekulationskontrolle ist und dass man durch die Untersuchung des Nominalismus als universitärer Spekulationskontrolle das Typische des Nominalismus ans Licht bringt ${ }^{3}$ ?

Und was heißt 'universitäre Spekulationskontrolle'? Ist sie die interne Kontrolle, die die Institution 'Universität' anwendet, um zu gewährleisten, dass bestimmte Regeln befolgt werden, die sie bei der Deutung von Texten als notwendig erachtet ${ }^{4}$ ? Hat sich der Nominalismus mit dieser Aufgabe identifiziert, und dient er als Wachhund über die wissenschaftliche Integrität an der Universität? Aber dann stellt sich sofort die Frage, ob es sich überhaupt mit der Institution Universität verträgt, darüber zu wachen, wie nach Wissen gesucht wird. Denn wie die Universität in unserer Zeit strebte schon die mittelalterliche als universitas

3. Die Frage, was Nominalismus im Mittelalter sei, wird diskutiert in C. NORMORE, "The Tradition of Mediaeval Nominalism», in: J. WIPpel (ed.), Studies in Medieval Philosophy, Washington 1987, pp. 201-217, und W. J. COURTENAY, "In Search of Nominalism. Two Centuries of Historical Debate», in: A. Maierù/R. ImbaCH (eds.), Gli Studi di filosofia medievale tra otto e novecento. Contributo a un bilancio storiografico, Rom 1991, pp. 241-233. Einen Blick auf die moderne Fragestellung bietet D. M. ARMSTRONG, Nominalism and Realism. Universals and Scientific Realism 1, Cambridge 1978.

4. Beispiel einer solchen Kontrolle ist das sogenannte Nominalismus-Statut, das die Pariser Artes-Fakultät im Jahre 1340 herausgab. Dieses Statut verbietet eine Vorgehensweise, nach der die Wahrheit oder Unwahrheit von Aussagen lediglich aufgrund logischer Kriterien (suppositio personalis) bestimmt wird und schreibt vor, die Absicht des Autors (intentio auctoris) zu berücksichtigen. Nach der älteren Literatur richtete sich dieses Statut gegen den Ockhamismus. Die neuere Forschung (Kaluza) deutet das Statut anders und erwägt Johannes Buridan als möglichen Autor. Zur Bedeutung dieses Statuts und zur Frage nach der Urheberschaft, siehe Z. KALUZA, "Les sciences et leurs langages. Note sur le statut du 29 décembre 1340 et le prétendu statut perdu contre Ockham», in: L. BIANCHI (ed.), Filosofia e teologia nel trecento. Studi in ricordo di Eugenio Randi, Louvain-la-Neuve 1994, pp. 197-258, bes. 242-245, und J. M. M. H. THIJSSEN, "The Crisis over Ockhamist Hermeneutic and its Semantic Background: the Methodological Significance of the Censure of December 29, 1340", in: C. MArmo (ed.), Vestigia, Imagines, Verba. Semiotics and Logic in Medieval Theological Texts (XIIth-XIVth Century), Turnhout 1997, pp. 371392. 
magistrorum et scolarium nach Unabhängigkeit ${ }^{5}$. In der Theologie, die sich auf die Offenbarung stützt, ist es nicht verwunderlich, dass eine solche Kontrolle stattfindet. Der Mensch darf sich nicht über Gott stellen. Hier gilt, um mit einem häufig zitierten Wort Augustins zu sprechen: «nobis autem ad certam regulam loqui fas est» ${ }^{6}$. Aber trifft dies auch auf die Philosophie zu? Ist nicht in der Philosophie das menschliche Denken selbst der Maßstab? Kann Spekulationskontrolle in der Philosophie etwas anderes bedeuten als 'Halte dich an die menschliche Vernunft und an nichts anderes als die menschliche Vernunft ${ }^{7}$ ? Wenn dies tatsächlich so ist, ist dann der Nominalismus die Philosophie der Philosophie, also die Philosophie, die die Philosophie an ihre eigenen philosophischen Ausgangspunkte erinnert?

\subsection{Selbstbild der Nominalisten und der Vertreter der via moderna}

Diese Fragen sind vielleicht abstrakt und spekulativ. Mit Blick auf die historische Wirklichkeit aber scheint sich der spätmittelalterliche

5. Zur Idee der Universität siehe J. Verger, Les Universités au Moyen Age, Paris 1973, bes. p. 48, und W. RÜEGG (ed.), Geschichte der Universität in Europa, Bd. 1: Mittelalter, München 1993, bes. pp. 24-48. Weiterführendes Quellenmaterial bietet auch der Art. «Philosophie V. Institutionelle Formen», Historisches Wörterbuch der Philosophie 7, Darmstadt 1989, coll. 795-848, bes. 808-819 (G. SCHRIMPF).

6. Augustin, De civitate Dei, lib. 10, cap. 12 (eds. B. Dombart/A. Kalb, Opera omnia 14/1, Turnhout 1955, p. 297) (CCSL 47). In seiner Kritik an der Mystik des Johannes Ruusbroec hebt Johannes Gerson diesen Satz hervor. Vgl. seinen zweiten Brief an den Kartäuser Batholomäus Clantier (geschrieben zu Paris, April-Juni 1408), abgedruckt in P. GlorieuX (ed.), Euvres complètes 2, Paris 1960, pp. 97-103, bes. 97. Zu dieser Auseinandersetzung siehe A. COMBES, Essai sur la Critique de Ruysbroeck par Gerson, 3 vol., Paris 1945-1972, und G. WARNAR, Ruusbroec. Literatuur en Mystiek in de Veertiende Eeuw, Amsterdam 2003, p. 392 (Reg. s. v. 'Gerson').

7. Eine solche Überzeugung, dass der Philosoph als Philosoph nur der Vernunft Rechenschaft schuldet, findet sich in Albertus Magnus, Metaphysica, (ed. B. GeYer), Opera omnia 16/2, Münster 1964, lib. 11, tract. 2, cap. 10, p. 495b: «Philosophi enim non est aliquid fingere et non dicere, nisi quod per rationem potest ostendi». Albert spricht hier von den 'praecipui peripateticorum'. Zum Philosophieverständnis Alberts mit Angabe von weiteren Stellen siehe L. STURLESE, Die deutsche Philosophie im Mittelalter. Von Bonifatius bis zu Albert dem Großen (748-1280), München 1993, pp. 324-388, bes. 344. Zum Postulat eines autonomen Vernunftgebrauchs, wie es im dreizehnten Jahrhundert an der Pariser Artes-Fakultät erhoben wurde, siehe F.-X. PutallaZ und R. IMBACH, Professione Filosofo. Sigieri di Brabante, Mailand 1998, und (mit Bezug auf die Verurteilung von 1277) D. PICHÉ, La Condamnation Parisienne de 1277, Paris 1999, bes. pp. 189-194. 
Nominalismus in der Gestalt der via moderna tatsächlich als 'Philosophie der Philosophie' profiliert zu haben. In einem Vortrag aus dem Jahre 1469 leitet der Nominalist Stephan Hoest das Wort 'moderna' von 'modus' und 'mensura' ab, um anzudeuten, dass die via moderna der neue Maßstab jeglicher philosophischer Reflexion sei und deshalb die Grenzen der Erkenntnis auf dem Gebiet der Philosophie bestimme: «in campo philosophico diversarum limites veritatum constituens» ${ }^{8}$. Er ist kein Einzelfall. Im Namen der Philosophie als studium veritatis et non erroris kritisiert der Nominalist Bartholomäus von Usingen im Jahre 1497 den dogmatischen Gebrauch der Werke des Aristoteles. Philosophen, die Aristoteles und nicht die Wahrheit selbst für den Maßstab in der Philosophie halten, machen sich ihm zufolge als amatores veritatis lächerlich: "non sunt philosophiae, id est veritatis amatores, sed philosophorum derisores» ${ }^{9}$.

\subsection{Vorgehensweise}

Diese Zeugnisse bieten uns wesentliche Informationen über das Selbstbild der spätmittelalterlichen Nominalisten. Allerdings lassen sie sich nicht einfach interpretieren. Studiert man sie näher, stellen sich sofort etliche Fragen. Stephan Hoest betrachtet nicht nur Ockham, Buridanus und Marsilius, sondern auch Heinrich von Gent und Johannes Duns Scotus als principes der via moderna ${ }^{10}$. Dass Heinrich von Gent und Johannes Duns Scotus zur via moderna gerechnet werden, ist

8. Es handelt sich hier um eine Rede, die Stephan Hoest als Vizekanzler der Heidelberger Artes-Fakultät bei der Verleihung der Lizenz an die Kandidaten der via moderna ausgesprochen hat. Sie ist abgedruckt in STEPHAN HOEST, Reden und Briefe. Quellen zur Geschichte der Scholastik und des Humanismus im 15. Jahrhundert (ed. F. BARON), München 1971, pp. 168-170.

9. BARTHOLOMÄUS VON USINGEN, Quaestio de quiditate quantitatis continae in disputatione de quolibet Erffordie A.D. 1497 determinata (ed. H.-U. WÖHLER), in: Bochumer philosophisches Jahrbuch für Antike und Mittelalter 6 (2001), pp. 137-195, bes. 187. Über den Nominalismus des Bartholomäus von Usingen informiert S. LALLA, Secundum viam modernam. Ontologischer Nominalismus bei Bartholomäus Arnoldi von Usingen, Würzburg 2003 (mit Bibliographie).

10. Stephan Hoest, Reden und Briefe (ed. F. BARON), p. 176: «(...) ut de principibus moderne invencionis sileam, Henrico scilicet de Gandavo, acutissimo et philosopho et theologo, Johanne Scoto, qui ob sui generalis prerogativam acuminis meruit subtilis cognominari (...)». Hoest vergleicht hier die via moderna mit einer moderna inventio, einer neuen Erfindung. 
merkwürdig und widerspricht dem Bild moderner Forscher vom Nominalismus. Dieser Befund bedarf somit einer Erklärung. Aber es geht noch weiter. Bartholomäus von Usingen beanstandet, wie gesagt, den unkritischen Gebrauch des Aristoteles. Jedoch spricht er in diesem Zusammenhang nicht nur als amator veritatis, sondern auch als Beschützer der Theologie. Nach seiner Überzeugung stehen einige Lehren des Aristoteles in offenem Widerspruch zur Offenbarung. Sein Vorbehalt gegen Aristoteles ist ein Vorbehalt gegen diejenigen, die Aristoteles uneingeschränkt als Autorität in der Theologie heranziehen wollen ${ }^{11}$. Bestimmt der Nominalismus also auch die Grenzen des Wissens auf dem Feld der Theologie? Bei näherem Studium zeigt sich, dass die Zeugnisse des Stephan Hoest und des Bartholomäus von Usingen tatsächlich die historische Wirklichkeit des Nominalismus widerspiegeln, jedoch anders, als man es auf den ersten Blick vermuten würde.

Um die aufgeworfenen Fragen zu beantworten und einen Interpretationsansatz der historischen Wirklichkeit des spätmittelalterlichen Nominalismus zu entwickeln, der auch die Aussagen des Stephan Hoest und des Bartholomäus von Usingen einzuordnen vermag, möchte ich zunächst zeigen, wie die Begriffe 'Nominalismus', 'moderni' und 'via moderna' im späten Mittelalter verwendet wurden. In einem folgenden Schritt werde ich dann anhand repräsentativer Beispiele für die Anwendung der Logik in der Theologie auf die Methodologie der moderni eingehen. Abschließend komme ich auf die Eingangsfragen zurück, indem ich auf der Grundlage der Ergebnisse die Bedeutung des Nominalismus als Spekulationskontrolle erörtern werde.

11. Bartholomäus VON Usingen, Quaestio de quiditate (ed. H.-U. WÖHLER), p. 187: «Sed hic quidam proterve insultant dicentes blasphemare illos in scolis philosophorum, qui negant Aristotilem. Et quod plus est, dicunt Aristotilem nusquam sensisse contra fidem catholicam. Quibus respondens moneo quatinus obmutescant et Aristotilem diligentius legant». Seine Kritik am dogmatischen Gebrauch des Aristoteles ist keine Kritik an der Philosophie. Nach Bartholomäus von Usingen entspricht die Wahrheit der Philosophie der Wahrheit der Offenbarung, wenn auch die Philosophie nicht immer imstande ist, die Wahrheit der Offenbarung vollständig zum Ausdruck zu bringen, da sie sich lediglich auf die natürliche Vernunft (lumen naturale) stütze. Dazu mit Quellenverweisen S. LALLA, Secundum viam modernam (nt. 9), pp. 266-273. 


\section{Begriffsgeschichtliche Analyse}

\section{1. 'Nominalismus'}

Untersucht man den Gebrauch des Begriffs 'Nominalismus' im vierzehnten und fünfzehnten Jahrhundert, treten einige bemerkenswerte Besonderheiten hervor. Diejenigen Theologen und Philosophen, die in der modernen Forschung als die Begründer des spätmittelalterlichen Nominalismus gelten, wie Ockham, Buridanus und Marsilius, bezeichnen sich nie selbst als 'Nominalisten'. Erst seit Beginn des fünfzehnten Jahrhunderts lässt sich der Begriff als Name für die Vertreter der via moderna ausmachen. Im dreizehnten und vierzehnten Jahrhundert hingegen wurde er nur in einem historischen Sinn verwendet, und zwar um auf bestimmte Theologen des zwölften Jahrhunderts zu verweisen. Es handelt sich bei den als 'nominales' Angesprochenen hauptsächlich um die sogenannten 'vocales', aber auch um diejenigen Theologen, die die Theorie der unitas nominis verteidigt hatten und dadurch zu ihrem Beinamen gelangt waren ${ }^{12}$.

\subsubsection{Vorgeschichte}

Studiert man die Werke von Ockham, Buridanus und Marsilius, bietet sich nicht die geringste Grundlage, ihre Autoren als vocales oder als Anhänger der Theorie der unitas nominis einzustufen ${ }^{13}$. Damit stellt sich die Frage, weshalb der Begriff 'Nominalist' mit einem Mal von

12. Die diesbezüglichen Quellen sind zusammengetragen in Y. IWAKUMA/s. EBBESEN, "Logico-Theological Schools from the Second Half of the 12th Century: A List of Sources», in: Vivarium 30 (1992), pp. 173-210. Siehe auch Y. IWAKUMA, "Vocales or early Nominalists», in: Traditio 47 (1992), pp. 37-111. Einigen Quellen des zwölften Jahrhunderts zufolge ist zwischen 'nominales' und 'vocales' zu unterscheiden. Dazu A. DE LIBERA, Art. "Universaux», in: C. Gauvard/A. DE Libera/M. ZinK (eds.), Dictionnaire du Moyen Age, Paris 2002, coll. 1417-1419, bes. 1419.

13. Nach Ockham, Buridanus und Marsilius sind die Universalien nicht lediglich als allgemeine Laute zu verstehen, wie die vocales behauptet hatten, sondern auch und sogar vor allem als natürliche Zeichen im menschlichen Denken. Ebensowenig vertreten sie die Theorie der unitas nominis, derzufolge das Erkenntnisobjekt das unveränderliche enuntiabile ist, beispielsweise das 'te currere', und die enuntiabilia diversorum temporum wie 'te currere', 'te cucurisse' und 'te esse cursurum' alle dasselbe bedeuten. Für Ockham, Buridanus und Marsilius jedoch ist das Objekt der Erkenntnis der Satz (propositio), nicht das enuntiabile oder das dictum propositionis. Siehe dazu die noch immer grundlegende Studie von G. Nuchelmans, Theories of the Proposition. Ancient and Medieval Conceptions of the Bearers of Truth and Falsity, Amsterdam 1973, bes. pp. 195-202 und 243-254. 
Theologen und Philosophen dafür herangezogen wurde, Denker wie Ockham, Buridanus oder Marsilius zu benennen. Um diese Frage beantworten zu können, ist zu berücksichtigen, dass die Auffassungen der vocales und der nominales im Laufe des zwölften und dreizehnten Jahrhunderts als communiter non approbatae, falsae und sogar haereticae angesehen wurden und dass diese negativen Urteile im späten vierzehnten Jahrhundert bekannt waren. Die wichtigsten Quellen in diesem Zusammenhang waren die Werke von Anselm von Canterbury und Bonaventura. So betonte Anselm, dass die vocales mit ihren Auffassungen über die Universalien eine Bedrohung für die Theologie bedeuteten, und nannte sie deshalb 'dialecticae haeretici'14. In ähnlicher Weise urteilte Bonaventura über die Anhänger der Theorie der unitas nominis, die nominales. Diese seien nicht in der Lage, einen Unterschied zu machen zwischen incarnatio futura und incarnatio facta. Denn für die nominales ist das Erkenntnisobjekt in beiden Fällen das 'incarnationem esse'. Bonaventura zufolge ist es aber dringend erforderlich, hier sorgfältig zu unterscheiden, da man andernfalls unverzüglich in Irrtümer gerate: «errans circa verum complexum quantum ad differentiam temporis simpliciter iudicatur haereticus» ${ }^{15}$.

\subsubsection{Das fünfzehnte Jahrhundert}

$\mathrm{Zu}$ den ersten Texten, in denen der Begriff 'Nominalist' in seiner neuen, spätmittelalterlichen Bedeutung auftaucht, gehört Contra modernos des Heymericus de Campo. Dieses Textstück bildet den Anfang des Tractatus problematicus und wurde im Laufe des fünfzehnten Jahrhunderts mehrfach abgeschrieben und überarbeitet. Aus diesen Bearbeitungen geht hervor, dass die Darstellung des Heymericus nicht nur aufgegriffen, sondern auch verschärft wurde ${ }^{16}$. Heymericus

14. Anselm von Canterbury, Epistola de incarnatione Verbi, cap. 1 (ed. F. S. Schmitt, Opera omnia 2, Stuttgart-Bad Cannstatt 1968, pp. 9-10): «(...) illi utique nostri temporis dilectici, immo dilecticae haeretici, qui non nisi flatum vocis putant universales esse substantias (...) a spiritualium quaestionum disputatationum sunt exsufflandi».

15. Bonaventura, Commentaria in quatuor libros Sententiarum, Opera omnia 3, Quaracchi 1887, lib. 3, dist. 24, art. 1, q. 3, 515b-516a. Siehe auch ibid., 516b: «(..) positio illa Nominalium, quae dicebat, enuntiabilia diversorum temporum esse unum, communiter non approbatur, immo falsa est (...)».

16. Der Tractatus problematicus wurde um 1422 in Köln geschrieben und 1496 bei Johannes Landen gedruckt. Der Druck und die meisten Handschriften überliefern den ganzen Traktat. In der Handschrift München, Universitätsbibliothek, $2^{\circ}$ Cod. ms. 49, 
ist in Contra modernos außergewöhnlich kritisch gegenüber den Auffassungen Ockhams, Buridans und des Marsilius. Ihre Theorien über die Universalien bedeuten das Ende der Wissenschaft als einer objektiven Disziplin, so behauptet er. Das Objekt der Wissenschaft sei das Allgemeine. Bestreite man, dass Universalien außerhalb des menschlichen Denkens existieren, sei keine Wissenschaft von der äußeren Wirklichkeit mehr möglich. Wissenschaft beschäftige sich dann nicht mehr mit realen Gegenständen, sondern nur noch mit menschlichen Vorstellungen und bloßen Wörtern. Um dieser Kritik Nachdruck zu verleihen, bezeichnet er die Verteidiger der Theorien Ockhams, Buridans und des Marsilius als 'litterales' und 'superficiales' 17.

Ein anonymer Bearbeiter von Contra modernos griff diese Darstellung auf, bezeichnete die litterales als 'nominales' und 'terministae' und fügte hinzu, dass diejenigen, die die Universalien nicht als Dinge, sondern als termini betrachten, wie es die nominales tun, nach Anselm als dialecticae haeretici zu gelten haben ${ }^{18}$. Die Auffassungen

fol. 1ra-54rb, fehlt jedoch Contra modernos. Über die Handschriftenlage informiert ausführlich P. RUTTEN, "Contra occanicam discoliam modernorum. The So-Called 'De universali reali' and the Dissemination of Albertist Polemics against the 'via moderna'", in: Bulletin de Philosophie Médiévale 45 (2003), pp. 131-165.

17. Heymericus De CAMPO, Contra modernos, in: Tractatus problematicus, Köln 1496 (Hain *4302), fol. 2v: «Taliter dicentes non sunt professores peripateticae veritatis, sed sunt epicurei litterales et superficiales sequentes condemnatam parisius occanicam discoliam cum collegiis suis, scilicet Buridano et Marsilio». Auskunft über die Quellen der Begriffe 'epicurei litterales et superficiales' gibt Z. KALUZA, «Le De universali reali de Jean de Maisonneuve et les epicuri litterales», in: Freiburger Zeitschrift für Philosophie und Theologie 33 (1986), pp. 469-516, bes. 502-507. In seiner Kritik an den 'litterales' und 'superficiales' stützt Heymericus sich auf die Ausführungen seines Lehrers Johannes de Nova Domo, wie letzterer sie in seinem Capitulum de universali reali dargelegt hatte. Dieses Capitulum ist ediert in A. WeILER, "Un traité de Jean de Nova Domo sur les Universaux», in: Vivarium 6 (1968), pp. 108-154, bes. 126-130. Eine überarbeitete Fassung ist herausgegeben in H. WeLs, Aristotelisches Wissen und Glauben im 15. Jahrhundert. Ein anonymer Kommentar zum Pariser Verurteilungsdekret von 1277 aus dem Umfeld des Johannes de Nova Domo. Studie und Text, Amsterdam 2004, xc-civ. Die Authentizität dieses Capitulum, welches den ersten Teil einer von Weiler unter dem Titel De universali reali edierten und als Ganzes Johannes de Nova Domo zugeschriebenen Schrift darstellt, wurde von Kaluza (ibid., pp. 471472 und 510-511) angezweifelt, jedoch zu unrecht, wie Rutten (ibid., pp. 142-148) hat zeigen können. Dagegen ist dem dritten Teil, der bisher als echt betrachtet wurde, die Autorschaft des Johannes abzusprechen. Er ist eine Bearbeitung und Erweiterung von Contra modernos des Heymericus, wie Rutten (ibid., pp. 148-155) aufgrund des handschriftlichen Befundes nachgewiesen hat.

18. Diese anonyme Bearbeitung ist zu Unrecht als dritter Teil des Traktats De universali reali von Johannes de Nova Domo ediert in A. WeILER, Un traité de Jean de Nova Domo 
Ockhams, Buridans und des Marsilius wurden im vorliegenden Fall vom Bearbeiter vereinfacht und in einer Weise dargestellt, dass sie exakt solchen Theorien glichen, die von den großen Theologen der Vergangenheit als Irrtümer zurückgewiesen worden waren, was jedoch dem Inhalt und dem Charakter der Schriften der fraglichen Autoren überhaupt nicht gerecht wird. Anhand von Beispielen wie dem vorausgehenden wird ersichtlich, dass der spätmittelalterliche Nominalismus eine Erfindung von Gegnern der via moderna ist, die auf diesem Wege die via moderna in Misskredit bringen wollten ${ }^{19}$. Erst im Laufe des fünfzehnten Jahrhunderts wurde der Begriff 'Nominalismus' dann auch von Nominalisten selbst gebraucht, und zwar als Synonym für die via moderna und in Formulierungen wie secundum viam nominalium.

\section{2. 'Via moderna'}

Damit stellt sich die Frage, wie es nun mit dem Begriff 'via moderna' steht. Der Begriff 'via' bedeutet 'Methode' und verweist auf den

(nt. 17), pp. 131-152, bes. 131-133. Die Stelle, in der Anselm von 'dialecticae haeretici' spricht, wurde von Johannes Wyclif in De veritate sacrae scripturae, lib. I, cap. 8 (ed. R. BuDDENSIEG, London 1905, p. 169), zitiert und kam über die Schriften des Wyclif nach Prag, wo sie bereits in den 1380er Jahren aufgegriffen und in den Debatten über die Universalien eingesetzt wurde, jedoch ohne Verbindung mit den Begriffen 'nominales' oder 'terministae'. Siehe dazu D. Trapp, "CLM 27034. Unchristened Nominalism and Wycliffite Realism at Prague in 1381", in: Recherches de Théologie ancienne et médiévale 24 (1957), pp. 320-360, bes. 355. Ein ausdrücklicher Bezug zur Universalienlehre des Marsilius wurde von Hieronymus von Prag in einer Disputation hergestellt, die er 1406 in Heidelberg abgehalten hatte, wie die Akten des Wiener Prozesses bezeugen. Vgl. Processus iudiciarius contra Jeronimum de Praga habitus Viennae a. 1410-1412 (ed. L. KLICMAN, Prag 1898, pp. 13 und 14sq.): "Interrogatus de fama [sc. Jeronimi de Praga, MH] dicit [magister Conradus de Hildeshaim, $\mathrm{MH}$ ] quod communiter a magistris Haidelberge tenebatur suspectus, ita quod eum eciam vitaverint propter dicta in disputacione sua proposita et concessa et propter opinionem universalium realium, quam tenuit in uno correlario suo, quod magistrum Marsilium et supradictos [dixit fuisse] hereticos in loica et philosophia ex eo, quia non tenerent universalia realia». Eine ähnliche Aussage, jedoch ohne Erwähnung von Namen, ist bezeugt im ersten Artikel seiner Quaestio de universalibus (ed. J. SEDVLAK, Studie a Texty k Zivotopisy Husovu, Olomouc 1915, pp. 215-224, bes. 221). Der Begriff 'nominales' wird in seiner Positio de universalibus (ibid., pp. 229-258) verwendet.

19. In seiner Positio de universalibus spricht Hieronymus von Prag fast immer von der 'via nominalium', um die Lehre seiner Gegner zu bezeichnen. Siehe J. SEDVLAK, Studie a Texty k Zivotopisy Husovu (nt. 18), p. 248: "Et quia ista via Nominalium dicit se tenere in hac materia [sc. de universalibus, $\mathrm{MH}$ ] sensum Aristotelis de directo, ideo primo adducam auctoritates Aristotelis cum suo Commentatore contra viam istam, ut videatur aperte, quam sit aliena via Nominalium ab eorum sentencia». 
Universitätskontext sowie auf die Weise, wie die Texte des Aristoteles und des Lombardus studiert wurden ${ }^{20}$. In der Verbindung mit 'moderna' taucht dieser Begriff im späten vierzehnten Jahrhundert auf und ist seit dem Beginn des fünfzehnten Jahrhunderts ein üblicher Terminus an den Universitäten ${ }^{21}$. Der Begriff 'moderna' wiederum bezieht sich auf die moderni, eine bestimmte Gruppe von Philosophen und Theologen, die sich von den sogenannten 'antiqui' durch die Form der Auseinandersetzung mit den Texten des Aristoteles und des Lombardus unterschieden.

\subsubsection{Umbruch im frühen vierzehnten Jahrhundert}

Wenngleich die Begriffe 'antiqui' und 'moderni' von der Zeit abhängen (moderni wurden nach einer bestimmten Zeit antiqui), lässt sich doch beobachten, dass sich die konkrete Bedeutung schon zu Beginn des vierzehnten Jahrhunderts festigte. Scotus markiert in diesem Zusammenhang den Umschlagspunkt. Im vierzehnten Jahrhundert bezeichnete man die Autoren vor ihm als 'antiqui', die Autoren nach ihm hingegen als 'moderni"22. Den Anlass für diese Unterscheidung bildeten Veränderungen hinsichtlich der Methode, die von den mittelalterlichen Gelehrten als etwas Besonderes registriert wurden und die von durchschlagender Bedeutung für die weitere Geschichte der Philosophie und Theologie an den Universitäten sein sollten. Untersucht

20. Dazu mit Quellenbelegen M. J. F. M. HoENEN, «Via antiqua and via moderna in the Fifteenth Century: Doctrinal, Institutional, and Church Political Factors in the Wegestreit», in: R. L. FRIEDMAN/L. O. NIELSEN (eds.), The Medieval Heritage in Early Modern Metaphysics and Modal Theory, 1400-1700, Dordrecht 2003, pp. 9-36, bes. 13-14.

21. F. EHRLE, Der Sentenzenkommentar des Peters von Candia des Pisaner Papstes Alexanders V. Ein Beitrag zur Scheidung der Schulen in der Scholastik des vierzehnten Jahrhunderts und zur Geschichte des Wegestreits, Münster 1925, pp. 281-342. In diesem für die Forschung noch immer bedeutenden Anhang sind mehrere Aktenstücke abgedruckt, die über den Wegestreit an den Universitäten informieren. Wichtige Materialien sind auch gesammelt in A. L. Gabriel, "Via Antiqua' and 'Via Moderna' and the Migration of Paris Students and Masters to the German Universities in the Fifteenth Century», in: A. ZIMMERMANN (ed.), Antiqui und Moderni. Traditionsbewußtsein und Fortschrittsbewußtsein im späten Mittelalter, Berlin 1974, pp. 439-493.

22. W. J. Courtenay, "Antiqui and Moderni in Late Medieval Thought», in: Journal of the History of Ideas 48 (1987), pp. 3-10. Die Bedeutung von 'moderni' als Zeitgenossen geht zurück bis auf antike Quellen der nachklassischen Zeit. Siehe hierzu CH. T. Lewis und CH. ShOrT, A Latin Dictionary, New York 1879, Oxford 1991, s.v. 'modernus'. 
man die Texte aus dieser Zeit, dann fällt vor allem auf, dass seit Beginn des vierzehnten Jahrhunderts die kritische Methodenreflexion immer mehr Aufmerksamkeit genießt. Diese kritische Reflexion äußert sich besonders deutlich in den Kommentaren zu den Sentenzen des Lombardus. Nicht länger stehen die von Petrus vorgegebenen Themen im Mittelpunkt, sondern die eigenen systematischen Fragen, die ausführlich diskutiert werden und den Text des Lombardus in den Hintergrund drängen ${ }^{23}$.

$\mathrm{Zu}$ beachten ist jedoch, dass zu dieser Zeit nicht nur diejenigen moderni Methodenreflexionen anstellen, die später als 'nominales' bezeichnet werden, wie sich am Beispiel der sich durchsetzenden distinctio formalis plastisch zeigt. Während Ockham ihre Anwendung außerhalb der Trinität vehement ablehnt, verteidigt sein im fünfzehnten Jahrhundert als Scotist und Realist bezeichneter Zeitgenosse Franciscus de Mayronis sie aus methodologischen Gründen, wenn er behauptet, dass das menschliche Denken sie auch in anderen Bereichen zwingend voraussetzen müsse. Bestreite der Theologe das Bestehen einer distinctio formalis, dann trete er in Widerspruch zu seinem Selbstverständnis als Wissenschaftler (contra conscientiam suam ${ }^{24}$.

Bemerkenswert ist, dass im fünfzehnten Jahrhundert exakt diese kritische Haltung, die aus einem geschärften Methodenbewusstsein hervorgeht, als Umschlagspunkt aufgefasst wird. Dionysius der Kartäuser hält die distinctio formalis für eine novitas, die keinerlei Fundament in der Tradition besitze, sondern von Scotus einzig und allein aufgrund von methodologischen Erfordernissen entwickelt worden

23. Dazu R. L. Friedman, "The Sentences Commentary, 1250-1320. General Trends, The Impact of the Religious Orders, and the Test Case of Predestination», in: G. R. Evans (ed.), Mediaeval Commentaries on the Sentences of Peter Lombard. Current Research 1, Leiden 2002, pp. 41-128, bes. 90sq. Der wichtigste Ort für die Erörterung von Methodenfragen waren die Prologe, die in der Zeit sehr umfangreich gestaltet wurden, wie die Kommentare von Scotus und Ockham exemplarisch belegen.

24. François de Meyronnes - Pierre Roger, Disputatio (1320-1321), (ed. J. BARBet), Paris 1961, p. 191. Zu Ockham siehe R. SCHÖNBERGER, "Realität und Differenz. Ockhams Kritik an der distinctio formalis», in: W. VOSSENKUHL/R. SCHÖNBERGER (eds.), Die Gegenwart Ockhams, Weinheim 1990, pp. 97-122. Die Position von Franciscus de Mayronis habe ich dargestellt in "Formalitates phantasticae. Bewertungen des Skotismus im Mittelalter», in: M. PICKAVÉ (ed.), Die Logik des Transzendentalen, FS für Jan A. Aertsen, Berlin 2003 (Miscellanea Mediaevalia 31), pp. 337-357, bes. 347-355. 
sei $^{25}$. Dies erklärt, weshalb Scotus von Stephan Hoest in seinem Vortrag aus dem Jahre 1469 als 'modernus' etikettiert wurde. Aber Scotus ist bekanntlich kein Nominalist. Zwischen 'modernus' und 'Nominalist' ist also zu differenzieren.

2.2.2. Aufspaltung der moderni an der Schwelle zum fünfzehnten Jahrhundert

Zwar reflektieren sowohl Scotus als auch Ockham kritisch auf ihre Methoden, jedoch sind die Ausgangspunkte, auf die sie sich jeweils stützen, grundverschieden. Dies lässt sich an ihrer Theorie der Univozität exemplarisch veranschaulichen. Beide sind der Meinung, dass von einer Univozität zwischen Gott und den Geschöpfen zu sprechen sei, und sie kritisieren in diesem Zusammenhang Thomas von Aquin. Wo aber Scotus auf eine metaphysische Analyse des ens inquantum ens zurückgreift, ist Ockhams Ansatz ein semantischer. Es sei möglich, so argumentiert Ockham, einen Begriff zu bilden, der auf Gott und auf die Geschöpfe verweise, und damit gebe es ein Deo et creaturae aliquid commune univocum ${ }^{26}$.

Bis etwa 1370 blieben die Unterschiede hinsichtlich der Ausgangspunkte eine Angelegenheit der einzelnen Philosophen und Theologen, die miteinander in Diskussion traten. Niemand sah in diesen Debatten eine Spaltung zwischen zwei verschiedenen Richtungen, derjenigen der Realisten und derjenigen der Nominalisten. Erst im letzten Viertel des vierzehnten Jahrhunderts änderte sich die Situation. Auf einmal wurde das Problem der verschiedenen Ausgangspunkte nachdrücklich thematisiert. Zuerst geschah dies in Oxford und durch John Wyclif. Er kritisiert die sogenannten 'doctores signorum', die zur Analyse der Schrift eine Methode verwendeten, die nach seiner Überzeugung bei der Schriftauslegung nichts verloren hatte ${ }^{27}$. Die Prinzipien der Analyse müssten in der Schrift selbst fundiert sein, da die Schrift

25. Dionysius DER KARTÄUSER, In librum primum Sententiarum, Opera omnia 19, Tournai 1902, dist. 2, q. 2, p. 173bCD.

26. Wilhelm VON OCKHAM, Scriptum in librum primum Sententiarum. Ordinatio, lib. 1, dist. 2, q. 9 (responsio auctoris) (eds. S. BROWN/G. GÁL, Opera theologia 2, St. Bonaventure, New York 1970, p. 306). Zu Scotus siehe L. HonNefELDER, Ens inquantum ens. Der Begriff des Seienden als solchen als Gegenstand der Metaphysik nach der Lehre des Johannes Duns Scotus, Münster Westfalen 1979, p. 466 (Reg.) s.v. 'univocatio, univocatio entis'.

27. Wyclif verwendet den Begriff 'doctores signorum' in seinem Tractatus de universalibus, cap. 2 (ed. I. J. Mueller, Oxford 1986, p. 53). Zum geistigen Umfeld in Oxford siehe 
die gesamte Wahrheit umfasse, so argumentiert er. In diesem Zusammenhang schneidet er ein Thema an, das von dieser Zeit an nicht mehr von der Tagesordnung weichen wird, nämlich das Universalienproblem $^{28}$. Wyclif zufolge spricht die Schrift explizit davon, dass Gott universalia realia geschaffen habe, namentlich in der Genesis: "creavit Deus cete grandia omnem animam viventem atque motabilem quam produxerant aquae in SPECIES suas et omne volatile secundum GENUS suum ${ }^{29}$. Seiner Meinung nach steht damit fest, dass jeder Philosoph und Theologe, der nach der Wahrheit der Schrift suche, derartige universalia realia als Ausgangspunkte seiner Analyse nehmen müsse ${ }^{30}$.

Zwar wurde das Universalienproblem schon diskutiert, bevor Wyclif mit seiner Theorie hervortrat ${ }^{31}$. Mit seiner fundamentalen Stellungnahme jedoch provozierte er seine Gegner, indem er die theologische Relevanz der Problematik zeigte. Bestreite man die Existenz von universalia realia, dann negiere man die Wahrheit der Schrift, so Wyclif. Darüber hinaus vertrat er die Auffassung, dass ein angemessener Begriff der universalia den Schlüssel zum Lösen aller Probleme auf dem Gebiet der Trinität und der Inkarnation biete: «non enim est aliqua fallacia in materia de trinitate vel incarnatione, quin potest proportionaliter detegi in materia de universalibus» ${ }^{32}$. Das Universalienproblem gelangte so in den Mittelpunkt der Debatten über die wissenschaftliche Methodologie. Hier endlich scheiden sich die Wege der moderni des vierzehnten Jahrhunderts. Die Verteidiger der universalia

J. I. CATto, «Wyclif and Wycliffism at Oxford, 1356-1430», in: J. I. Catto/R. Evans (eds.), The History of the University of Oxford 2, Oxford 1992, pp. 175-261.

28. Dazu ausführlicher mit Quellenverweisen M. J. F. M. HoENEN, "Jean Wyclif et les universalia realia. Le débat sur la notion de virtus sermonis au Moyen Age tardif et les rapports entre la théologie et la philosophie», in: J.-L. SOLÈrE/Z. KALUZA (eds.), La servante et la consolatrice. La philosophie dans ses rapports avec la théologie au Moyen Age, Paris 2002, pp. 173-192.

29. Johannes Wyclif, De veritate sacrae scripturae, cap. 8 (ed. cit., Band 1, p. 169; Hervorhebungen MH). Vgl. Gen. 1, 21. Ähnliche Gedanken mit Verweisen auf die Schrift artikuliert er in seinem Tractatus de universalibus, cap. 8 (ed. cit., pp. 167-171).

30. JOHANNES WyCLIF, De veritate sacrae scripturae, cap. 8 (ed. cit., Band 1, p. 182).

31. Einen historischen Überlick gibt A. DE LibERA, La Querelle des Universaux. De Platon à la Fin du Moyen Age, Paris 1996.

32. JOHANNES WYCLIF, De veritate sacrae scripturae, cap. 8 (ed. cit., Band 1, p. 169). In seinem Tractatus de universalibus, cap. 8 (ed. cit., p. 175) und cap. 15 (ed. cit., p. 357), macht Wyclif ähnliche Behauptungen. 
realia stützten sich auf die Autoritäten aus der Zeit vor Scotus (die antiqui), um ihre Position zu stärken, und legten so das Fundament für die via antiqua. Ihre Gegner setzten den eingeschlagenen Weg fort und machten weiterhin Gebrauch von Werken der Autoren aus der Zeit nach Scotus, wie Ockham, Adam Wodeham und Robert Holkot, die sich gegen die Existenz von universalia realia ausgesprochen hatten ${ }^{33}$.

\subsubsection{Philosophie, Theologie und der Wegestreit}

Über die besprochenen Zusammenhänge hinaus hat die Theologisierung des Universalienproblems noch eine weitere wichtige Konsequenz: Sie richtete die Methodendiskussion auf die Frage nach dem Verhältnis von Philosophie und Theologie. Betrachtet man universitäre Zeugnisse aus dem fünfzehnten Jahrhundert, fällt auf, wie häufig die Debatten zwischen der via antiqua und der via moderna mit diesem Problem verknüpft sind. Im Zentrum dieser Zeugnisse steht immer wieder die Frage, wie theologische Irrtümer vermieden werden können ${ }^{34}$. Laut Marsilius von Inghen, der schon zu Beginn des fünfzehnten Jahrhunderts als Urheber der via moderna galt, sind philosophische und theologische Argumente voneinander zu unterscheiden, da der Mensch aufgrund philosophischer Erwägungen nicht immer entscheiden könne, ob ein Argument wahr sei, sondern nur, ob es gültig sei. Als Beispiel führt er die creatio ex nibilo an. Aufgrund des Prinzips ex nihilo nibil fit, dem jeder Philosoph zustimmen müsse, sei der Schluss zwingend, dass die Welt aus etwas geschaffen wurde. Dies aber stehe in kontradiktorischem Widerspruch zur Wahrheit des Glaubens, der zufolge die Welt aus dem Nichts geschaffen wurde. Halte der Philosoph an seinen eigenen Prinzipien fest, führe er den Theologen auf die falsche Spur. Der Philosoph denke ex puris naturalibus, während der Theologe sich auf die Offenbarung stütze. Philosophie und Theologie seien deshalb sorgfältig auseinander zu

33. Über diese Debatten und deren Bezüge zu den Positionen des dreizehnten und frühen vierzehnten Jahrhunderts informieren die Ausführungen von A. D. Conti in JOHANNES SHARPE, Quaestio super universalia (ed. A. D. CONTI), Florenz 1990, bes. pp. 211-336.

34. Siehe die Aktenstücke, die bei F. EHRLE, Der Sentenzenkommentar (nt. 21), pp. 281-342, bes. 282 (Köln), 292 (Leuven), 326 (Paris), 334 (Ingolstadt), abgedruckt sind. Wichtige Materialien sind auch zugänglich gemacht in H. WELS, Aristotelisches Wissen und Glauben im 15. Jahrhundert (nt. 17). 
halten ${ }^{35}$. Die Anhänger der via antiqua lehnen diese Einschätzung indes ab. Ihrer Meinung nach gehören Philosophie und Theologie vielmehr untrennbar zusammen. Überdies sei die Philosophie in der Lage, aufgrund ihrer eigenen Prinzipien die Theologie zu unterstützen. Um ihren Standpunkt zu untermauern, verweisen sie auf Thomas von Aquin, der als Theologe auf das Werk des Aristoteles zurückgegriffen habe ${ }^{36}$.

Exemplarisch für diese Debatte ist eine Disputation, die im Jahre 1480 an der Universität Köln gehalten wurde. In dieser Disputation steht ein modernus Verteidigern der via antiqua gegenüber ${ }^{37}$. Thema ist die göttliche Trinität, genauer: die Frage, ob die göttliche Einheit mit der Dreiheit der göttlichen Personen vereinbar sei. Der modernus behauptet, dass er sich zur Beantwortung dieser Frage nicht auf Aristoteles stützen könne, sondern lediglich zu referieren vermöge, was die Kirche festgelegt habe, da die Trinität der menschlichen Vernunft unzugänglich sei: "plura hic oportet dicere quae intellectus non capit» ${ }^{38}$. Damit sind die antiqui nicht einverstanden. Sie halten Aristoteles für den besten Leitfaden, um das Problem der Trinität zu durchleuchten. Die Debatte endet in einem heillosen Durcheinander. Der Schreiber der Handschrift bringt dies prägnant zum Ausdruck, indem er gleich am Anfang der Disputation konstatiert: «modernus, qui posuit Colonie inconsueta, bene scobatus fuit» ${ }^{39}$. Über die

35. Siehe Marsilius von Inghen, Quaestiones super quattuor libros Sententiarum, Straßburg 1501, Nachdruck Frankfurt am Main 1966, lib. 1, q. 42, art. 2, fol. 177va: «Hoc [sc. creationem esse Deo possibilem, $\mathrm{MH}$ ] ex lumine naturali aeque facile est probare sicut asinum volare, cum oppositum creationis sit principium omnium philosophorum, scilicet nihil fieri nisi ex subiecto praesupposito». Die natürliche Vernunft (lumen naturale) nimmt ihren Ausgang von der sinnlichen Wahrnehmung. Darum ist sie in vielen Fällen (in multis) nicht imstande, die Wahrheit des Glaubens (veritas catholica) zum Ausdruck zu bringen, wie Marsilius, ibid., fol. 179ra hervorhebt.

36. Siehe den Text aus nt. 63 weiter unten.

37. Diese Disputation ist erhalten in der Handschrift Frankfurt, Stadt- und Universitätsbibliothek, Ms. Praed. 102. Dazu G. M. LÖHR, Die theologischen Disputationen und Promotionen an der Universität Köln im ausgehenden 15. Jahrhundert, Leipzig 1926, bes. p. 61. Der modernus wird am Rand der Handschrift, am Anfang der Disputation, fol. 86r, als 'Magister Iohannes Alen, modernus' identifiziert. Johannes Alen hatte an der ArtesFakultät zu Erfurt studiert, einer Hochburg der via moderna. Siehe H. Keussen, Die Matrikel der Universität Köln, Band 1: 1389-1475, Bonn 1928, Nachdruck Düsseldorf 1979, n. 306 (1465), 43, p. 732. Eine Edition dieser Disputation bereite ich derzeit vor.

38. Ibid., fol. $87 \mathrm{r}$.

39. Ibid., fol. 86r. 
Hintergründe dieses Durcheinanders lässt die Darstellung der Disputatio keinen Zweifel entstehen. Es geht um die Autorität des Aristoteles in der Theologie, die der modernus bestreitet: "assumpsit aliud, quia dixit eum negare omnia fundamenta Aristotelis» ${ }^{40}$. Wir befinden uns hier auf dem Höhepunkt des Wegestreits.

\subsection{Vorläufige Bilanz}

Als historische Strömung tritt der Nominalismus erst in Zeugnissen des fünfzehnten Jahrhunderts hervor. Seine Wurzeln sind jedoch älter und reichen zurück bis zum Beginn des vierzehnten Jahrhunderts, in dem eine lebhafte Debatte über die wissenschaftliche Methode einsetzt. Sämtliche Theologen und Philosophen aus dieser Zeit werden als 'moderni bezeichnet. Im letzten Viertel des vierzehnten Jahrhunderts ändert sich die Lage. Das Auftreten Wyclifs zwingt zu einer fundamentalen Positionsbestimmung auf dem Gebiet der universalia und hinsichtlich des Verhältnisses von Philosophie und Theologie. Infolge dessen entstehen an den Universitäten zwei verschiedene Strömungen im Bereich der wissenschaftlichen Methodologie, deren eine unter dem Namen 'via moderna' den Weg fortsetzt, der zu Beginn des vierzehnten Jahrhunderts eingeschlagen wurde, während die andere unter dem Namen 'via antiqua' auf Autoritäten aus dem dreizehnten Jahrhundert zurückgreift. Indem sie die via moderna nunmehr als nominalistisch und damit als communiter non approbata etikettiert, versucht die via antiqua, an den Universitäten Boden zu gewinnen ${ }^{41}$.

\section{Die Methodologie der moderni}

Im weiteren Verlauf dieses Beitrags wird die Methodologie der moderni eingehender untersucht und herausgearbeitet, wie es zur oben erwähnten fundamentalen Spannung zwischen Philosophie und Theologie

40. Ibid., fol. $87 \mathrm{r}$.

41. Im sechzehnten Jahrhundert, als sich der Wegestreit gelegt hat, tritt in den scholastischen Texten die alte Bedeutung von 'moderni' als Zeitgenossen wieder hervor, ohne Verbindung mit dem Nominalismus. Dazu M. TAVUZZI, "Moderni, Nominales and Terministae in the Compendium logicae isagogicum of Chrysostomus Javelli O.P. (ca. 1470-1538)», in: A. Lombato (ed.), Littera, sensus, sententia. Studi in onore del Prof. Clemente J. Vansteenkiste, Mailand 1991, pp. 573-592, bes. 575. 
kam. Zu diesem Zweck soll zunächst die Anwendung der Logik in der Trinitätslehre ins Auge gefasst werden ${ }^{42}$. Die herausgegriffenen Beispiele lassen erkennen, wie moderni wie Durandus de St. Pourçain, Wilhelm von Ockham, Robert Holkot und Marsilius von Inghen die Grenzen der wissenschaftlichen Methodologie freilegten und einer Theologie Raum boten, die der Philosophie gegenüber kritisch eingestellt war. Auf dieser Grundlage ist dann zu den Fragen zurückzukehren, die am Anfang dieses Beitrags aufgeworfen wurden.

\subsection{Der Begriff 'Deus'}

Im Zentrum des ersten Beispiels steht die Bedeutung des Begriffs 'Deus'. Im dreizehnten Jahrhundert wird der Begriff 'Deus' von mehreren Theologen als ein Name aufgefasst, der in der logischen Argumentation wie ein Eigenname (terminus discretus) verwendet wird, da er auf eine Form verweist, die nicht mitteilbar ist - nämlich die göttliche Wesenheit. Diese Position vertritt unter anderen Bonaventura. Er versteht 'Deus' als 'terminus habens formam non multiplicabilem' und erläutert die Bedeutung dieses Begriffes in verneinenden Sätzen, indem er Beispiele mit Eigennamen wie 'Petrus non currit' und 'Non Petrus currit' anführt ${ }^{43}$. Seit dem frühen vierzehnten Jahrhundert ändert sich die Deutung des Begriffs 'Deus'. Er gilt seither als ein allgemeiner Begriff (terminus communis), da er für jede der drei göttlichen Personen zu stehen vermag. Eine Verwendung nach den Regeln

42. Zu dieser Thematik siehe H. G. Gelber, Logic and the Trinity. A Clash of Values in Scholastic Thought, 1300-1335, Ann Arbor, Michigan 1981 (Ph.D. Thesis 1974, University of Wisconsin) und M. H. SHANK, Unless You Believe. You Shall Not Understand: Logic, University, and Society in Late Medieval Vienna, Princeton 1988. Besonders eingehend hat sich auch A. Maierù mit Fragen der trinitarischen Logik im späten Mittelalter befasst. Von seinen Veröffentlichungen sei erwähnt «Logica e theologia trinitaria nel commento alle Sentenze attribuito a Petrus Thomae», in: J. Jolivet/Z. KAluZA/A. DE LiberA (eds.), Lectionum varietates. Hommage à Paul Vignaux (1904-1987), Paris 1991, pp. 177-198, mit Angaben zu seinen weiteren Studien auf p. 186 (nt. 4) und p. 187 (nt. 16).

43. Siehe Bonaventura, Commentaria in quatuor libros Sententiarum, Opera omnia 1, Quaracchi 1882, lib. 1, dist. 4, art. un., q. 1, p. 98a: "Tertia regula est, quod termino habenti formam non multiplicabilem non differt praeponere et postponere negationem. Unde non differt dicere 'Petrus non currit' et 'Non Petrus currit'». Und ibid., p. 98b: "Cum enim iste terminus 'Deus' dicat formam immultiplicabilem, non differt ei praeponere negationem et postponere». Die von Bonaventura angeführte Regel setzt die Verneinung des Prädikats (postponere negationem) mit der Verneinung des Satzes (praeponere negationem) gleich, wenn das Subjekt des Satzes als Eigenname (terminus discretus) zu verstehen ist. 
der Eigennamen wird abgelehnt, etwa von Durandus de St. Pourçain, der sich gegen die von Bonaventura vertretene Position wendet ${ }^{44}$. Diese neue logische Deutung des Begriffes 'Deus' hat weitreichende Folgen für die Analyse von Sätzen wie p 'Deus generat Deum' und $\neg$ p 'Deus non generat Deum'. Nach der alten Auffassung kann nur einer der beiden Sätze wahr sein. Denn bei Eigennamen schließen Negation und Affirmation einander aus: 'Socrates ambulat' und 'Socrates non ambulat' können nicht beide zugleich wahr sein. Bei einem Allgemeinbegriff jedoch ist dies anders, wie das Beispiel 'Homo ambulat' und 'Homo non ambulat' zeigt. Zwischen diesen beiden Sätzen besteht kein logischer Widerspruch, wenn 'homo' im einen Fall etwa auf Sokrates verweist, im anderen auf Platon. Aufgrund solcher Erwägungen behaupten moderni wie Durandus und Ockham, dass sowohl $\mathrm{p}$ 'Deus generat Deum' als auch $\neg \mathrm{p}$ 'Deus non generat Deum' wahr sein können, da im bejahenden Satz p 'Deus' für 'Deus Pater' steht, im verneinenden Satz $\neg \mathrm{p}$ für 'Deus Filius' oder 'Deus Spiritus Sanctus' 45 .

Infolge solcher Entwicklungen im Bereich der Logik ist jedoch eine gefährliche Ambiguität in die Theologie eingedrungen, die es zuvor nicht gab. Wenn nämlich jemand die Behauptung aufstellt, 'Deus non generat Deum', lässt sich mit logischen Mitteln nicht mehr zweifelsfrei entscheiden, ob er orthodox oder heterodox ist. Er affirmiert die Trinität im orthodoxen Sinn, wenn das Subjekt 'Deus' auf den 'Deus Filius' verweist, aber er negiert sie, wenn das Subjekt 'Deus' für den 'Deus Pater' steht. Für Autoren des dreizehnten Jahrhunderts bestand

44. Siehe Durandus DE ST. POURÇAIN, In Sententias commentariorum libri IIII, Venedig 1571, Nachdruck RidGEWOOD, New Jersey 1964, lib. 1, dist. 4, q. 2, n. 10, fol. 29ra: «(...) Deus non habet rationem termini discreti, sed communis inquantum tribus personis convenit, ratione cuius communitatis aliquis diceret et bene quod differt praeponere et postponere negationem». Durandus kritisiert hier die Gleichsetzung von Verneinung des Prädikats und Verneinung des Satzes bei der Verwendung des Begriffes 'Deus', die Bonaventura noch verteidigt hatte. Über die Datierung des Sentenzenkommentars des Durandus, der in drei verschiedenen Versionen überliefert ist, siehe R. L. FrIEDMAN, "The Sentences Commentary 1250-1320» (nt. 23), pp. 71-72. Die gedruckte Version, aus der hier zitiert wird, bietet die dritte Version, entstanden zwischen 1317 und 1327.

45. Durandus de ST. Pourçain, In Sententias (ed. cit.), lib. 1, dist. 4, q. 2, n. 13, fol. 29rb: «Et ideo cum generare conveniat Patri, ista potest verificari 'Deus generat'. Et cum non conveniat Filio nec Spiritui Sancto, potest ista verificari 'Deus non generat' (...).», Wilhelm VON OcKham, Scriptum in librum primum Sententiarum. Ordinatio, lib. 1, dist. 4, q. 1, (ed. G. ETZKORN, Opera Theologica, 3, St. Bonaventure, New York, 1977, pp. 14-15). 
dieses Problem nicht. Aufgrund seines anderen Logikverständnisses konnte Bonaventura beispielsweise noch problemlos äußern, dass $\neg \mathrm{p}$ 'Deus non generat Deum' falsch sei, da p 'Deus generat Deum' wahr $\operatorname{sei}^{46}$.

Im vierzehnten Jahrhundert vermochte die Logik somit nicht länger, eine 'Garantie' für die Orthodoxie theologischer Aussagen zu bieten. Zeitgenössische Theologen lassen in dieser Hinsicht keinen Zweifel aufkommen. So behauptet Durandus in seinem Sentenzenkommentar, dass der Satz $\neg \mathrm{p}$ 'Deus non generat Deum' seiner logischen Form nach wahr sei (potest de virtute sermonis verificari), dass es sich aber dennoch empfehle, diesen Satz nicht zu verwenden, da er Unerfahrene zu dem falschen Schluss verleiten könne, dass in Gott überhaupt keine generatio vorkomme und folglich auch keine drei Personen in ihm bestünden: "non recipitur, ne sit occasio erroris in imperitis ex hoc credentibus quod omnino non sit generatio in divinis» ${ }^{47}$. Eine vergleichbare Reaktion zeigt Ockham. Der Satz $\neg \mathrm{p}$ 'Deus non generat Deum' sei gemäß den Regeln der Logik (secundum artem logicam) ohne weiteres gültig $(\text { formalis })^{48}$. Da er aber zu Irrtümern verleite, sei er als unwahr anzusehen: "propter haereticos (...) potest dici quod haec (sc. propositio) est falsa» ${ }^{49}$. Unwahr (falsa) sei er nicht im «logischen» Sinne, sondern im "theologischen»: Man nenne den Satz unwahr, obgleich er dies eigentlich nicht sei, um den Glauben zu beschützen.

\subsection{Der Unterschied zwischen den göttlichen Personen}

Das zweite Beispiel stammt aus einer etwas späteren Zeit und findet sich in den Werken des Adam Wodeham, Robert Holkot und Marsilius von Inghen. Es betrifft die Frage, ob der Unterschied zwischen

46. Bonaventura, Commentaria in quatuor libros Sententiarum (ed. cit.), lib. 1, dist. 4, art. un., q. 1, p. 98b: «(...) alium modum habet supponendi [sc. terminus 'Deus', MH] in affirmativa quam in negativa [sc. propositione, $\mathrm{MH}$ ], quia in affirmativa erat locutio vera pro Patre, in negativa vero non potest esse veritas (...)». Bonaventura verweist zur Begründung dieser Auffassung auf die von ihm zuvor erwähnte 'tertia regula'.

47. Durandus De ST. PourÇAin, In Sententias (ed. cit.), lib. 1, dist. 4, q. 2, n. 13, fol. 29rb.

48. Wilhelm von Ocкham, Scriptum in librum primum Sententiarum (ed. cit.), lib. 1, dist. 4, q. 1, p. 14.

49. Ibid. 
den göttlichen Personen unendlich groß oder unendlich klein sei ${ }^{50}$. Gemäß dem üblichen Sprachgebrauch (der usualis locutio) sei dieser Unterschied unendlich klein, so die besagten moderni, da Gott ungeteilt sei ${ }^{51}$. Der Unterschied zwischen den göttlichen Personen berühre ja nicht die göttliche Einheit. Es handele sich also um einen Unterschied, der so klein ist, dass er gar nicht kleiner sein könne, denn wäre eine Steigerung möglich, wäre Gott nicht ungeteilt. Dem Anschein nach stimme dieser Schluss mit dem Wissen der Logik überein. Und dennoch sei das genaue Gegenteil der Fall, so behaupten die moderni. Ziehe man nämlich die logische Bedeutung der Wörter in Betracht (die vis vocis), dann gelte, dass der Unterschied (distinctio) zwischen den drei göttlichen Personen unendlich groß sei. Denn der Unterschied (distinctio) zwischen drei Dingen sei logisch gesprochen nichts anderes als diese drei Dinge selbst. Im Fall der göttlichen Personen aber sei jedes dieser Dinge unendlich, da jede der drei Personen Gott sei und dieser wiederum unendlich. Damit sei jedoch der Unterschied zwischen den Personen unendlich groß und nicht unendlich klein, wie es dem alltäglichen Verständnis (usualis intellectus) nach zu behaupten sei ${ }^{52}$. Robert Holkot gibt zu erkennen, dass die Regeln der Logik hier nicht nur dem üblichen Sprachgebrauch widersprechen, sondern auch in Konflikt geraten mit der gängigen Meinung der Theologen, die stets betont haben, dass der Unterschied unendlich klein sei (solet dici a doctoribus quod distinctio personalis est minima $)^{53}$.

50. Siehe dazu ausführlich meinen «Virtus Sermonis and the Trinity: Marsilius of Inghen and the Semantics of Late Fourteenth-Century Theology», in: Medieval Philosophy and Theology 10 (2001), pp. 151-171, bes. 165-169, mit einer Besprechung der Auffassungen von Adam Wodeham, Robert Holkot und Marsilius von Inghen.

51. So etwa Adam Wodeham, Super quattuor libros Sententiarum (Abbreviatio Henrici de Oyta), Paris 1512, lib. 1 dist. 33, q. 8, fol. q6va. Über die Schriften und die Theologie des Adam Wodeham gibt Auskunft R. WOOD, «Introduction» in: R. WoOD/G. GÁL (eds.), ADAM WODEHAM, Lectura secunda in librim primum Sententiarum 1, St. Bonaventure, New York, 1990, pp. 5*-38*.

52. Adam Wodeham, Super quattuor libros Sententiarum (ed. cit.), lib. 1 dist. 33, q. 8, fol. q6va: «(...) haec distinctio sit infinita, quia quaelibet persona est infinita (...). Sed ad usualem intellectum dicendum est longe aliter (...)». Neben usualis intellectus spricht Adam Wodeham, ibid., auch von usualis locutio und vulgatus modus loquendi. Für Robert Holkot und Marsilius von Inghen siehe Hoenen, "Virtus Sermonis and the Trinity» (nt. 50), pp. 165-169.

53. Robert Holkot, In quatuor libros Sententiarum quaestiones, Lyon 1518, Nachdruck Frankfurt am Main 1967, lib. 1, q. 5, fol. f1vb (ad secundum). Über Holkot und 
Wenn die Logik allein ihren eigenen Prinzipien und Regeln folge, gerate sie somit auch in diesem Fall in Widerspruch zur Tradition.

\subsection{Logik und Trinität}

Das dritte Beispiel ist dem Sentenzenkommentar des Marsilius von Inghen entnommen. Er schrieb dieses Werk am Ende des vierzehnten Jahrhunderts, nachdem er schon eine große Anzahl an Schriften auf dem Gebiet der Logik publiziert hatte ${ }^{54}$. In seinem Kommentar geht er dementspechend oft auf logische Probleme ein, namentlich bei der Erörterung theologischer Probleme hinsichtlich der Trinität $t^{55}$. An einer dieser Stellen behauptet er, dass laut den moderni philosophi et logici in einem wahren Satz dem Prädikat das Wort 'tantum' ohne Veränderung der Bedeutung des Satzes hinzugefügt werden könne. So sei beispielsweise der Satz $\mathrm{p}_{2}$ 'Socrates est tantum Socrates' gleichbedeutend mit dem Satz $\mathrm{p}_{1}$ 'Socrates est Socrates', da in beiden Sätzen Subjekt und Prädikat auf denselben Gegenstand verweisen und das Wort 'tantum' im Satz $\mathrm{p}_{2}$ lediglich besagt, dass das Prädikat 'Socrates' im Satz $\mathrm{p}_{2}$ identisch ist mit dem Prädikat 'Socrates' des Satzes $\mathrm{p}_{1}{ }^{56}$. Auf die Trinität angewendet heißt dies, dass der Satz $\mathrm{p}_{4}$ 'Filius est tantum Deus' gleichbedeutend ist mit dem Satz $\mathrm{p}_{3}$ 'Filius est Deus', da auch hier gilt, dass in beiden Sätzen Subjekt und Prädikat für denselben Gegenstand stehen, nämlich die göttliche Person des Sohnes. Marsilius lehnt den Satz $\mathrm{p}_{4}$ 'Filius est tantum Deus' jedoch ab, obwohl er nach den Regeln der Logik aus dem wahren Satz $\mathrm{p}_{3}$ folgt. Er begründet seine Ablehnung mit der Ambiguität des Satzes, da dieser sich auch so verstehen

seine Haltung in Bezug auf die Verwendung von Logik in der Theologie informieren F. Hoffmann, Die theologische Methode des Oxforder Dominikanerlehrers Robert Holcot, Münster 1972, und H. G. GeLBER, Exploring the Boundaries of Reason. Three Questions on the Nature of God by Robert Holcot OP, Toronto 1983.

54. Studien zu Leben und Werk des Marsilius sind zusammengestelt in M. J. F. M. HoeNEN und P. J. J. M. BAKKER (eds.), Philosophie und Theologie des ausgehenden Mittelalters. Marsilius von Inghen und das Denken seiner Zeit, Leiden 2000 (mit ausführlicher Bibliographie).

55. Die Trinitätslehre des Marsilius ist wenig studiert. Eine Ausnahme, wenn auch älteren Datums, ist W. MÖHLER, Die Trinitätslehre des Marsilius von Inghen. Ein Beitrag zur Geschichte der Theologie des Spätmittelalters, Limburg/Lahn 1949.

56. MARSILIUS VON INGHEN, Quaestiones super quattuor libros Sententiarum (ed. cit.), lib. 1, q. 24, art. 2 (secunda dubitatio), fol. 101 ra-rb. 
lasse, dass der Sohn nur göttlich und demnach nicht inkarniert sei. Damit aber trete man in einen offenen Widerspruch zur Orthodoxie. Würde der Theologe nicht nur den Satz $\mathrm{p}_{3}$, sondern auch den Satz $\mathrm{p}_{4}$ verteidigen, könnte er damit Dritte auf einen Irrweg leiten. Diese Ambiguität auszuschließen, ist die Logik nicht imstande. Nach Marsilius muss der Theologe in solchen Fällen seiner persönlichen Verantwortung als Lehrer gerecht werden und den Satz $\mathrm{p}_{4}$ 'Filius est tantum Deus' zurückweisen: "tutius est eam non concedere, ne erroris occasio simus» ${ }^{57}$.

\subsection{Tradition}

Diese Beispiele belegen, wie fundamental das Problem des Verhältnisses von Logik und Theologie für die moderni war. Die 'moderne' Linie wurde aufgegriffen durch Johannes Gerson, der ihr als Kanzler der Universität von Paris einiges Gewicht verlieh. Exemplarisch ist in diesem Zusammenhang der Traktat De duplici logica (1402), in dem er zwischen der Logik der Ethik und Theologie und jener der Physik und Metaphysik unterscheidet, die jeweils ihren eigenen Gesetzmäßigkeiten folgten ${ }^{58}$. Im ersten Teil seines De mystica theologia behandelt er ein weiteres Mal die Probleme der Logik im Bereich der Theologie und differenziert zwischen einer theologia scholastica und einer theologia mystica ${ }^{59}$. In der theologia scholastica würde auf dieselbe Weise räsoniert wie in der Philosophie (conformiter ad philosophicas disciplinas). Aber diese scholasticae exercitationes genügen nicht und können leicht zu Irrtümern führen, wie er bemerkt, es sei denn, die Theologen wissen exakt, was die von ihnen verwendeten Begriffe bedeuten. Weder

57. Diese Aussage steht in einem Zusammenhang, in dem Marsilius darauf hinweist, dass eine strikt angewendete logische Sprache dem Auftreten von Häresien Vorschub leisten könne, ibid., fol. 101rb: "Quamvis ergo de vi stricte locutionis talis sit vera [sc. 'Filius est tantum Deus'], quia tamen ea usi sunt haeretici, ut li 'tantum' excluderet humanitatem et sic faciat sensum falsum quem generare posset in simplicibus, ideo tutius est eam non concedere, ne erroris occasio simus. Ideoque etiam doctores nostri eam non admittunt, ne intelligatur male, quasi non sit homo (...) capiendo li 'tantum' ut excluderet aliam naturam a divinitate».

58. Johannes Gerson, De duplici logica (ed. P. Glorieux, Euvres complètes 3, Paris 1962, pp. 57-63, bes. 59): «Et hoc est quod quidam non irrationabiliter dicunt quod theologia suam habet logicam et modum loquendi proprium (...)».

59. Johannes Gerson, De mystica theologia, Consideratio 30 (ed. A. Combes, Lugano 1958, pp. 76-79). 
aus der Logik noch aus der Philosophie können sie diese Bedeutung gewinnen, sondern einzig aus der Tradition. Die Theologen müssen wissen, wie ihre wichtigsten Vorgänger (summi doctores) diese Begriffe gebraucht haben ${ }^{60}$. Halten sie stur an den Regeln der Logik fest, gleichen sie Kindern oder Redelustigen, die nicht wissen, worüber sie sprechen oder welchen Aussagen sie zustimmen: "pueri vel picae neque vere intelligentes de quibus loquntur neque de quibus affirmant» ${ }^{61}$.

Wie vorhin schon mit Verweis auf Wyclif erwähnt, schlossen sich nicht alle Theologen dieser Einschätzung an. Stattdessen versuchten manche, das Problem anders zu lösen, indem sie die philosophicae disciplinae umformten und sie auf diese Weise mit der Theologie in Übereinstimmung brachten. Probleme auf dem Gebiet der Trinität wollten sie durch das Studium der trinitarischen Struktur der Schöpfung lösen, wie Wyclif grundsätzlich formulierte: «contingit viatorem assentire trinitatem per trinitates repertas in creaturis» ${ }^{62}$. Hierfür griffen sie auf die Physik und die Metaphysik zurück. Diese Richtung wurde als via antiqua bezeichnet, da Autoren des dreizehnten Jahrhunderts als Vorbilder herangezogen wurden ${ }^{63}$. Beide Richtungen, die via moderna und die via antiqua, beriefen sich auf die Tradition. Aber wo es der via moderna um die Tradition der theologi approbati und um den vulgus modus loquendi ging, orientierte sich die via antiqua an der Tradition des Aristotelismus des dreizehnten Jahrhunderts oder versuchte

60. Ibid., p. 77.

61. Ibid., p. 77. In seiner Consolatio theologiae hebt Gerson die Beschränktheit der Philosophie mit Aussagen wie 'philosophia non cognovit' und 'philosophia non attingit' hervor. Siehe dazu M. S. Burrows, Jean Gerson and De Consolatione Theologiae (1418). The Consolation of a Biblical and Reforming Theology for a Disordered Age, Tübingen 1991, pp. $130-131$. p. 2).

62. Johannes Wyclif, Tractatus de Trinitate, cap. 1 (ed. A. D. BrecK, Colorado 1962,

63. Programmatisch bringen dies die Kölner Magister in einem Schreiben an die Kurfürsten (1425) zum Ausdruck, in dem sie mit Verweis auf Thomas und die Art und Weise, wie er Philosophie und Theologie verbunden habe, für den Erhalt der von ihnen eingeführten via antiquorum plädieren. Dieses Schreiben ist abgedruckt in F. EHRLE, Der Sentenzenkommentar (nt. 21), pp. 282-285 (Anhang von Aktenstücken), bes. 284: "Secundo dicimus quod Artium cum Facultate Theologie tam indissolubilis est connexio, quod per idem valere est, prohibere hujus doctrine usum in Artibus et in Theologia, et permittere in Theologia et in Artibus. Exempli gratia, Doctor Sanctus in omnibus summis suis utitur eisdem principiis, quibus usus et libros Philosophi exponendo, prout luce clarius constat cuilibet in ejus doctrina eruditio». 
- mit den kritischen Worte des modernus Petrus de Candia -, Aristoteles zu 'taufen' und in die Theologie zu integrieren (Aristotelem catholicum facere $)^{64}$.

\section{Ergebnisse}

Im Lichte der erzielten Befunde lassen sich abschließend die Fragen erörtern, die zu Beginn dieses Beitrags aufgeworfen wurden. 'Nominalismus als universitäre Spekulationskontrolle': Es wurde sichtbar, dass sich der mittelalterliche Nominalismus in einem bestimmten Sinne als 'universitäre Spekulationskontrolle' begreifen lässt. Er macht die Grenzen der mittelalterlichen Logik und damit der Scholastik überhaupt kenntlich, denn schon seit den zarten Anfängen der Scholastik bei Augustin und Alkuin ist es eine Aufgabe der Logik, Ambiguitäten auszuschließen ${ }^{65}$. Der Nominalismus zeigt, dass die Logik diese Aufgabe nicht alleine erfüllen kann, sondern dazu die Hilfe der Tradition benötigt. Er macht die Reichweite der menschlichen Rationalität transparent und schafft Raum für die Tradition, die den Gesetzen des vulgus modus loquendi folgt. Es ist daher kaum verwunderlich, dass der Nominalismus als non approbatus und als Zerstörer der Scholastik in die Geschichte eingegangen ist.

Das Aufzeigen von Grenzen ist nur die eine Seite der Medaille. Der Nominalismus folgt den Gesetzen der Logik und den Prinzipien der menschlichen Vernunft ex puris naturalibus. Er ist aufgrund seiner wissenschaftlichen Methodologie nicht bereit, hier einen Kompromiss einzugehen. Wo der Nominalismus von natürlicher Vernunft spricht, meint er eine Vernunft, die sich auf solche Prinzipien des menschlichen Denkens stützt, denen jeder zustimmen kann, ungeachtet der Ausgangspunkte des Glaubens. Die Ironie hinsichtlich des Nominalismus

64. Petrus de Candia macht diese Bemerkung am Anfang seines zweiten Sentenzenbuches bei der Behandlung der Frage, ob Gott etwas, das von ihm wesentlich verschieden ist, ewig habe hervorbringen können. Die Stelle ist zitiert in F. EHRLE, Der Sentenzenkommentar (nt. 21), p. 66: «Sed <quia> aliqui theologi volunt Aristotelem catholicum facere, dicunt quod nunquam fuit sua opinio, quod mundus eternaliter fuerit, sed potius oppositum, quod ex suius principiis nituntur probare (...)».

65. Dazu J. Pépin, Saint Augustin et la Dialectique, Villanova 1976, und J. Marenbon, From the Circle of Alcuin to the School of Auxerre. Logic, Theology and Philosophy in the Early Middle Ages, Cambridge 1981, bes. pp. 30-66. 
ist, dass diese strikte natürliche Haltung nicht akzeptiert wurde und Denker wie Ockham, Buridanus und Marsilius von Inghen nicht nur als dialecticae haeretici, sondern sogar als diabolice haeretici bezeichnet wurden ${ }^{66}$.

Marsilius von Inghen betont, dass die Theologie eine scientia pietatis sei, die sich in solchen Fällen nicht mit natürlichen Argumenten verteidigen müsse, in denen diese keine beweiskräftige Begründung für Glaubensfragen darstellten ${ }^{67}$. Dies bedeute nicht, dass die Verwendung der Philosophie in der Theologie ausgeschlossen sei. Die Philosophie und die Logik behalten eine wichtige Funktion in dem Bereich der Theologie, der von den natürlichen Grundlagen der Schöpfung und der Gotteslehre handelt, und besitzen dort Autorität ${ }^{68}$. Wo es aber um das gehe, was den Menschen übersteigt, nämlich um die Geheimnisse der Offenbarung, greifen sie Marsilius von Inghen zufolge als Beweismittel zu kurz und bergen das Risiko, die Gläubigen in die Irre zu führen.

Die Sicht, die Marsilius vertrat, kulminiert später in einer Notiz von Kaspar Peucer aus dem Jahre 1588, die den Titel Triplex aetas doctorum scholasticorum trägt. Er behauptet dort, dass mit der Aufnahme der aristotelischen Philosophie im frühen dreizehnten Jahrhundert die Theologie korrumpiert worden sei: «haec aetas, detestenda audacia et impudentia, pro scriptura et patrum doctrina invexit in ecclesiam Aristotelis philosophiam» ${ }^{69}$. Damit steht der mittelalterliche Nominalismus

66. So Hieronymus nach den Akten des Wiener Prozesses, siehe Processus iudiciarius contra Jeronimum de Praga habitus Viennae (ed. cit.), p. 13. Die Verwendung von 'diabolice' neben 'dialecticae' geht womöglich auf ein Spiel mit den lateinischen Abbreviaturen oder auf eine bestimmte handschriftliche Überlieferung der Texte von Anselm oder Wyclif zurück, denn 'dialectic $<a>e$ ', wie Anselm und Wyclif es verwenden, und 'diabolice' lassen sich, wenn abgekürzt, leicht verwechseln. Aus dem Apparat der Edition von Wyclifs De veritate Sacrae Scripturae geht hervor, dass diese Variante als handschriftliche Marginalie bezeugt ist; vgl. JoHANNES WyCLIF, De veritate sacrae scripturae (ed. cit.), p. 169, Codex Bodleianus 924 (B): «Nota quod negantes universalia realia sunt diabolici heretici secundum Anselmum».

67. MARSILIUS VON INGHEN, Quaestiones super quattuor libros Sententiarum (ed. cit.), lib. 1, dist. 45, art. 2, fol. 191vb: «Non est enim scientia sacra scientia praesemptionis vel logicalis superstitionis sed pietatis, quae non destruere debet simplices sed aedificare».

68. So begreift Marsilius von Inghen seinen Kommentar zur aristotelischen Metaphysik als eine Vorbereitung auf die Theologie. Vgl. G. RitTER, Marsilius von Inghen und die okkamistische Schule in Deutschland, Heidelberg 1921, p. 113 (nt. 3).

69. Diese Notiz wurde erstmals herausgegeben in D. CANTIMORI, Umanesimo e Religione nel Rinascimento, Turin 1975, pp. 108-109, nt. 30. Bereits Luther hatte sich in diesem Sinne über die Scholastik geäußert und damit die heftige Kritik der Pariser theologischen 
an der Wiege der modernen säkularisierten Wissenschaft, die sich bewußt von der Theologie abgrenzt. Die Ausgangspunkte der Theologie werden als persönliche Überzeugungen betrachtet, die sich nicht mit den Methoden der Wissenschaft untermauern lassen. Dem gegenüber gründet die Wissenschaft auf universellen Prinzipien, die jedermann nachvollziehen kann. Dass in unserer Zeit Theologen und Wissenschaftler Vorbehalte haben, miteinander in Diskussion zu treten, und den Glauben für eine private Angelegenheit halten, lässt sich als ein Echo dieser geschichtlichen Konstellation begreifen. Über persönliche Standpunkte könne man schließlich nicht sinnvoll diskutieren.

Fakultät eingeholt. Siehe Determinatio theologicae facultatis Parisiensis super doctrina Lutheriana per eam visa, Paris 1521 (nicht foliert), De philosophia et theologia scholastica [Propositio sexta Lutheri, $\mathrm{MH}$ ]: «Libidinem corrumpendae scripturae iam ferme trecentis annis [also seit dem Anfang des dreizehnten Jahrhunderts, $\mathrm{MH}$ ] patitur ecclesia incomparabili damno suo a doctoribus scholasticis'. Haec propositio est falsa, stulte et malitiose asserta». Siehe zu dieser Determinatio F. T. BOS, Luther in het oordeel van de Sorbonne. Een onderzoek naar ontstaan, inhoud en werking van de 'Determinatio' (1521) en naar haar verhouding tot de vroegere veroordelingen van Luther, Amsterdam 1974. 